\title{
UNA APROXIMACIÓN A LA REALIDAD DE LA SEXUALIDAD EN PACIENTES CON TRAUMATISMO CRANEOENCEFÁLICO (TCE)
}

\section{AN APPROACH TO REALITY OF SEXUALITY IN TRAUMATIC BRAIN INJURY PATIENTS}

\section{Esther Sánchez Raja*}

Asociación Nacional de Salud Sexual y Discapacidad (ANSSYD)*

E-mail: esanchezra@gmail.com 


\section{RESUMEN}

Los traumatismos craneoencefálicos (TCE) constituyen una causa importante de minusvalía neurológica persistente que afecta con mayor frecuencia a personas relativamente jóvenes, con diferentes grados de incapacidad durante muchos años. La respuesta y la expresión sexual, puede verse alterada por las secuelas físicas, neuropsicológicas, emocionales y conductuales. La atención y educación de estos aspectos, por parte del personal de Enfermería es fundamental.

\section{Objetivos General:}

Conocer el estado de salud sexual de las personas con TCE.

\section{Objetivo Específicos:}

1) Describir las características socio-demográficas y los datos relativos al accidente; 2) Describir si hay alteraciones sexuales tras el TCE; 3) Valorar las relaciones interpersonales anteriores y posteriores al TCE; 4) Conocer si durante la rehabilitación han recibido educación sexual.

\section{Metodología:}

Estudio descriptivo transversal. Datos: Cuestionario anónimo validado. Población: Asociaciones adscritas a la Federación Española de Daño Cerebral (FEDACE). 488 afectados. Contestan 70 pacientes $(14,3 \%, 52$ varones y 18 mujeres entre 16 y 46 años).

\section{Resultados:}

La causa más frecuente de TCE es accidente de tráfico. La mayoría después del TCE tienen afectada la sexualidad negativamente, no tienen pareja 
estable, han notado cambios a nivel de su vida social, afecto, autoestima y confianza, no han recibido educación sexual.

\section{Conclusiones:}

Después de un TCE, la sexualidad se ve alterada en la mayoría de los pacientes, siendo fundamental que el personal de enfermería aborde el tema y dé respuestas a estas necesidades y para ello, han de tener una formación previa en sexualidad, a fin de abordar y actuar de manera correcta ante tal situación.

\section{PALABRAS CLAVE:}

Traumatismo craneoencefálico, discapacidad, sexualidad, enfermería, disfunciones sexuales

\section{SUMMARY}

Title: AN APPROACH TO REALITY OF SEXUALITY IN TRAUMATIC BRAIN INJURY PATIENTS

Traumatic brain injury (TBI) is an important cause of persistent neurological disability that mostly affects young adults and includes varying degrees of long-term incapacity.

\section{General Objective:}

To assess the state of sexual health of people with TBI.

\section{Specific Objectives:}

To evaluate 1) patients sociodemographic characteristics and accident related data; 2) the existence of sexual dysfunction in patients who have 
suffered a TBI; 3) interpersonal relationships before and after TBI 4) if they have received sex education during rehabilitation.

\section{Design:}

Descriptive and transversal study. Data: Anonymous validated questionnaire. Study Subjects: Associations affiliated to the National Federation of Brain Damage (FEDACE). 488 affected. 70 final participants (14, 3\%, 52 men and 18 women, aged between 16 and 46 years).

\section{Results:}

Traffic accidents are the most common cause of TBI. After TBI, most patients have sexuality adversely affected, have no partner, noticed changes at the level of social life, affection, self-esteem and confidence and have not received sex education.

\section{Conclusions:}

After TBI, sexuality is altered in most patients, being necessary for nurses to address the issue and provide answers to these needs. Therefore, nurses essentially need prior training in sexuality, in order to address and properly act on such situation.

\section{KEY WORDS:}

Traumatic brain injury, disability, sexuality, nursery, sexual dysfunction, 


\section{INTRODUCCIÓN}

El daño cerebral adquirido (DCA) configura una realidad sanitaria y social de magnitud creciente. Según los últimos datos disponibles, en España residen 420.064 personas con DCA $^{1}$. Entre las principales causas destacan los accidentes cerebrovasculares (ACV) y los traumatismos craneoencefálicos ${ }^{2}$. Se estima que cada año se producen en nuestro país alrededor de 200 nuevos casos por cada 100.000 habitantes y año de TCE, de los cuales más de 2.500 quedarán con importantes secuelas ${ }^{2}$. El TCE se ha definido como una afectación del cerebro causada por una fuerza externa que puede producir una alteración o disminución del estado de conciencia, que conlleva una alteración de las habilidades cognitivas o del funcionamiento físico ${ }^{2}$. EI TCE se da con una mayor incidencia en hombres que en mujeres (ratio 3:1) y en jóvenes entre 15 y 25 años, seguido de dos picos de edad, la infancia y los mayores de 65 $a_{n ̃ o s}{ }^{3}$. En cuanto a la causa externa, los accidentes de tráfico constituyen el mayor porcentaje causal $(73 \%)$, seguido por las caídas $(20 \%)$ y lesiones deportivas (5\%), con acentuadas diferencias según el grupo de edad y sexo ${ }^{3-4}$.

La OMS clasifica los TCE según la escala de Glasgow para el coma (GCS) en traumatismos craneoencefálicos graves (GCS < 9), traumatismos craneoencefálicos moderados (GCS 9-13) y traumatismos craneoencefálicos leves (GCS 14-15). De los accidentes que se producen aproximadamente el $10 \%$ son graves, $10 \%$ moderados y $80 \%$ leves, aunque existe una gran variabilidad en la incidencia según los estudios realizados ${ }^{2-4}$. Tras la recuperación, las secuelas derivadas de estas lesiones (físicas, sensoriales, 
cognitivas y conductuales) son responsables del 40 por 100 de las nuevas grandes minusvalías ${ }^{2}$, por tanto, es fundamental que los usuarios con dichos traumatismos reciban la atención y tratamiento adecuados por parte del personal sanitario 5

De acuerdo la $\mathrm{OMS}^{6}$, la sexualidad es un aspecto central del ser humano, presente a lo largo de su vida. Abarca el sexo, las identidades y los roles de género, el erotismo, el placer, la intimidad, la reproducción y la orientación sexual. Se vivencia y se expresa a través de pensamientos, fantasías, deseos, creencias, actitudes, valores, conductas, prácticas, roles y relaciones interpersonales. La sexualidad forma parte de la personalidad del individuo manifestándose progresivamente a lo largo de la vida. En el ser humano no se limita a una necesidad fisiológica, sino que se amplía como una capacidad de comunicar, expresar, dar y obtener placer. La sexualidad está influida por la interacción de factores biológicos, psicológicos, sociales, económicos, políticos, culturales, éticos, legales, históricos, religiosos y espirituales. La OMS comienza a hablar de salud sexual integrando todos estos aspectos en la definición de trabajo propuesta en la conferencia realizada en Ginebra en $2006^{7}$ y que orienta también en la necesidad de atender y educar la sexualidad humana. Así entendida, la sexualidad y la afectividad constituyen una base sólida en el proceso de aprendizaje que dará como resultado una formación y desarrollo personal satisfactorio. La afectividad y la sexualidad deben desarrollarse satisfactoriamente en todos los individuos aunque estos no estén en plenitud de condiciones físicas y psíquicas ${ }^{8}$. 
Dada la gran complejidad de secuelas que pueden existir tras la lesión, los posibles efectos del TCE sobre la sexualidad de una persona pueden ser muy variados. Numerosos estudios realizados hasta la fecha coinciden en que los pacientes con TCE ven afectada su salud sexual, manifestando disminución del deseo, dificultad para alcanzar el orgasmo, dificultad para mantener la erección o problemas de eyaculación o lubrificación, entre otros ${ }^{9-12}$. Estos cambios pueden estar directamente relacionados con los daños estructurales y funcionales sufridos tras el $\mathrm{TCE}^{10}$ o ser secundarios al impacto de la medicación o reacciones psicológicas y emocionales como la depresión o ansiedad $^{12-14}$. Sin embargo, Aloni y Katz ${ }^{11}$ afirman que las causas y los efectos del funcionamiento sexual después de padecer un TCE son muy confusos y que la literatura actual no permite evaluar la contribución de cada problema a la disfunción sexual presentada, así como la incidencia actual de disfunción sexual tras el TCE. Además se conoce poco el cómo los afectados y/o sus parejas viven este proceso y cómo perciben las consecuencias del TCE sobre su salud sexual ${ }^{15}$.

En España no existen estudios específicos sobre las alteraciones en la salud sexual de los pacientes con TCE a excepción de alguna reseña realizada en el estudio elaborado por FEDACE para el Defensor del pueblo ${ }^{2}$, un estudio exploratorio de disfunciones sexuales en pacientes con $\mathrm{DCA}^{16}$, y el estudio piloto realizado sobre el efecto del TCEM en la salud sexual de las personas que lo padecen ${ }^{17}$, lo cual pone de manifiesto la pobre información existente en lo que se refiere al impacto que el TCE tiene sobre la calidad de vida (CV) y la 
salud sexual de estos pacientes.

La fundamentación teórica de las actividades desempeñadas por las profesionales de enfermería según los modelos teóricos clásicos que las sustentan ${ }^{18}$, nos lleva a afirmar que no se contempla ninguna intervención explícitamente encaminada a la atención de la dimensión sexual de las personas con excepción de los patrones funcionales de salud de Marjory Gordon $^{19}$. No es hasta finales de los años ochenta con la aparición de las taxonomías y nomenclaturas diagnósticas de enfermería que empiezan a describirse etiquetas diagnósticas referidas a la sexualidad y a las alteraciones que de ella se podían derivar.

De este modo, el objetivo de este trabajo es conocer como altera el TCE a la salud sexual de las personas afectadas desde un enfoque cuantitativo, con la finalidad de mejorar la atención, educación y cuidados que precisan estos pacientes tanto desde la perspectiva enfermera como interdisciplinar.

\section{MATERIAL Y MÉTODOS}

\section{Diseño del estudio}

Se realizó un estudio descriptivo de corte transversal durante los meses de Febrero a Julio de 2010. Para el estudio, se contactó con las 29 asociaciones adscritas a la Federación Española de Daño Cerebral (FEDACE). Siete asociaciones estuvieron interesadas en participar en el estudio: TRACE en Cataluña, ADACE-Lugo y ALENTO en Galicia, VIVIR (Almería) y ADACCEA 
(Jaén) en Andalucía, REHACER en Baleares, ADACE en Castilla la Mancha, contando con un total de 488 afiliados afectados por TCE. Se utilizó como criterio de inclusión ser personas menores de 65 años sin deterioro cognitivo.

Como técnica de recogida de datos se diseñó un cuestionario que incluye aspectos demográficos, datos relativos al accidente y datos relativos a sus hábitos sexuales antes y después del TCE. El cuestionario fue sometido a un proceso de validación por pacientes con TCE de la Asociación Catalana de Traumatismo Craneoencefálico y Daño cerebral adquirido (TRACE) en el cual se consideraron dos aspectos: 1) Si la pregunta estaba claramente planteada; 2) si el tema o asunto abordado era de importancia para el paciente.

Para el análisis estadístico se utilizó el paquete SPSS versión 15.0. Se realizó estadística descriptiva obteniéndose frecuencias y porcentajes. La estadística bivariada se realizó con las pruebas Chi cuadrado y $U$ de Mann Whitney según correspondiera. Los cálculos se realizaron con nivel de significación $p=0.05$.

\section{Consideraciones éticas.}

La participación de los sujetos encuestados en el estudio se realizó previa información de los objetivos del proyecto y el consentimiento informado de cada asociación y la autorización de la institución FEDACE para la realización del estudio. 


\section{RESULTADOS}

\section{Características socio-demográficas (sexo, edad) y datos relativos al accidente.}

Del total de participantes, contestaron a las encuestas 70 pacientes, representando el $14,3 \%$ de los asociados, 52 varones y 18 mujeres en edades comprendidas entre 16 y 46 años. El 94,5\% de las mujeres ( $17 / 18$ casos) y el $73 \%$ de los hombres (38/52 casos) declararon no tener pareja estable en el momento de la encuesta.

Los accidentes de tráfico representaron la principal causa de TCE (Figura 1) tanto en hombres $(80,8 \%, 42$ casos) como mujeres $(66,7 \%, 12$ casos) no existiendo diferencias entre sexos $(p=0.324)$.

\section{Sexualidad y actividad sexual antes y después del TCE.}

Al analizar la sexualidad de los pacientes (Figura 2), observamos que entre las mujeres existe una ligera disminución de la calidad de las relaciones antes y después del TCE aunque no de forma significativa $(p=0,308)$. El $50 \%$ frente al $66,7 \%$ de las mujeres afirma no haber tenido relaciones antes $y$ después de la lesión, respectivamente. Sin embargo, tal y como se observa en la figura 2, entre los hombres se produce una disminución importante en la calidad de las relaciones antes y después del TCE $(p=0,0001)$. El 63\% de los hombres afirma haber tenido relaciones satisfactorias antes de sufrir el TCE frente al 24,2\% tras el accidente.

Respecto a la actividad sexual, tanto en hombres como mujeres los datos revelan un aumento considerable de la masturbación y la disminución de la 
frecuencia de cualquier manifestación de la actividad sexual después del TCE (Figuras 3 y 4 ).

\section{Alteración de las relaciones interpersonales después del TCE.}

El $76,4 \%$ de las mujeres y el $78 \%$ de los hombres afirman haber sufrido una alteración negativa de sus relaciones interpersonales tras el TCE, no existiendo diferencias entre sexos $(p=0.789)$.

\section{Educación sexual en pacientes con TCE.}

El $44,4 \%$ de las mujeres encuestadas y el $53 \%$ de los hombres afirman utilizar métodos anticonceptivos $(p=0.492)$, siendo el preservativo el método más utilizado ( $83 \%$ casos), seguido de las pastillas anticonceptivas $(17 \%)$.

La inmensa mayoría de los participantes, señalaron tener dudas sexuales tras el TCE ( $89 \%$ de las mujeres y $92,3 \%$ de los hombres, $p=0.655)$. Entre las dudas más frecuentes podemos destacar: si el TCE afecta a las erecciones $(40 \%)$, si pueden tener hijos (34\%), si las relaciones sexuales pueden ser placenteras (12\%), o si se afecta la masturbación o el orgasmo (7\%), entre otros.

Finalmente, a la pregunta ¿Alguien te ha impartido educación sexual tras el TCE?, el $78 \%$ de las mujeres y el $63,5 \%$ de los hombres respondieron que no habían recibido ningún tipo de información sexual.

\section{DISCUSIÓN}

La sexualidad forma parte de la personalidad del individuo, manifestándose progresivamente a lo largo de la vida. En el ser humano no se 
limita a una necesidad fisiológica, sino que se amplia como una capacidad de comunicar, expresar, dar y obtener placer. Así entendida, la sexualidad y la afectividad constituyen una base sólida en el proceso de aprendizaje del individuo y deben desarrollarse adecuadamente en las personas aunque estas no estén en plenitud de condiciones físicas y psíquicas ${ }^{20}$. Sin embargo los profesionales de la salud prestamos poca atención a este aspecto, y aún menos a la educación y el tratamiento de las alteraciones sexuales en pacientes con algún tipo de discapacidad. Con este estudio se ha pretendido de abordar de forma preliminar las características de los pacientes españoles afectados de TCE.

Según los resultados, la población afectada de TCE es mayoritariamente masculina (75\%) y la principal causa de TCE en individuos adultos es el accidente de tráfico, tal y como ha sido descrito con anterioridad ${ }^{4}$.

Los cambios en el funcionamiento sexual son comunes después de un TCE. En los años 50 y 60, se realizaron los primeros estudios sobre poblaciones con TCE $21-23$, señalando ya entonces la existencia de trastornos de la conducta sexual posterior al traumatismo. A partir de los años 80 , Oddy y Humphrey ${ }^{24}$, demuestran que después del TCE aparecen problemas sexuales persistentes, y un aumento de la disminución de la frecuencia de relaciones coitales, datos afianzados por un estudio realizado recientemente 25 , y que coinciden con los datos aportados en este trabajo.

Respecto a la caracterización del tipo de alteraciones sexuales, Kosteljanetz et al.,26 observaron, en una muestra de 19 varones, una 
reducción de la libido (53\%) y la aparición de disfunción eréctil (42\%). Otros autores observaron alteraciones del deseo y disminución de la frecuencia de relaciones coitales ${ }^{13}$. Con relación al sexo femenino algunos autores ${ }^{14}$ han observado alteraciones del deseo, del orgasmo y la disminución de la frecuencia en las relaciones sexuales tras un TCE, así como una disminución importante en la lubrificación vaginal después del TCE ${ }^{12}$. En los resultados hallados en el estudio que aquí se presenta, también se manifiesta un aumento de las disfunciones sexuales tanto en varones como en mujeres así como una disminución en la frecuencia de las relaciones sexuales, aportando como dato novedoso un incremento de la masturbación tanto en varones como en mujeres como practica sexual más utilizada después de sufrir el TCE.

Simpson y Long ${ }^{27}$ evaluaron la educación sexual recibida por los pacientes tras padecer un TCE y los recursos de información utilizados por los mismos. Estos autores pudieron comprobar que las personas que habían podido aclarar sus dudas podían disfrutar más de su sexualidad. Sin embargo, el número de personas que recibieron alguna información durante el periodo rehabilitador fue muy pequeño $(10 \%)$ comparado con los que no lo recibían, tal y como ocurre en este trabajo. Tras el traumatismo, hay un aumento de las dudas sobre cómo será su sexualidad después del accidente, tanto en hombres como en mujeres y son muy pocos los que durante la etapa rehabilitadora han podido acceder a este tipo de información.

\section{CONCLUSIÓN}

Después del TCE, la sexualidad y la actividad sexual se ven alteradas en 
la mayoría de los pacientes. Esto justifica la necesidad por parte de los profesionales sanitarios de prestar atención a esta dimensión estableciendo la formación en sexualidad de forma reglada en los planes de estudio e integrando actividades de intervención en los protocolos clínicos de actuación.

\section{BIBLIOGRAFÍA}

1 El Daño Cerebral Adquirido (DCA) en España: principales resultados a partir de la Encuesta EDAD-2008. Boletín del Observatorio Estatal de la Discapacidad n03. 2011. p. 39-59.

2. Federación Nacional de Daño Cerebral adquirido (FEDACE). Daño cerebral sobrevenido en España, un acercamiento epidemiológico y sociosanitario. Coordinador: Bascones Serrano LM. Colaboradores: Quezada García MY, Fernández-Cid M, López Calle P, Tejero González M Madrid: Informe del Defensor del pueblo; 2005.

3. Bruns J, Hauser WA. The Epidemiology of Traumatic Brain Injury: A Review. Epilepsia. 2003; 44: 2-10

4. Orient F, Sevilla E, Guevara D, Terré R, Ramón S, Bernabeu M. Resultado Funcional al alta de los traumatismos craneoencefálicos graves ingresados en una unidad de daño cerebral. Rev Neurol 2004; 39: 901-906.

5. Federación Española de Daño Cerebral. Qué misión cumplimos. Disponible en: http://www.fedace.org/web/dano.php

6. Organización Mundial de la Salud (OMS). Defining sexuality. Ginebra, 1974.

7. Organización Mundial de la Salud (OMS). Defining sexual health: Report of a technical consultation on sexual health. Ginebra, 2006; p. 28-31.

8. Ernest R. Lemberg, G.,S, Davis F. A sexuality and the person with traumatic brain injury: a guide for families. Philadelphia: FA Davis Amazonas. 1993.

9. Sanders AM, Maestas KL, Pappadis MR, Sherer M, Hamond FM, Hanks R. Sexual functioning 1 year after traumatic brain injury: findings from a prospective traumatic brain injury model systems collaborative study. Arch Phys Med Rehabil. 2012; 93:1331-1337. 
10. Elliott ML, Biever LS. Head injury and sexual dysfunction. Brain Inj. 1996; 10:703-717.

11. Aloni, R, Katz S. A review of the effect of traumatic brain injury on the human sexual response. Brain Inj. 1999; 13:269-280.

12. Hibbard MR, Gordon WA, Flanagan S, Haddan L, Labinsky E. Sexual dysfunction after traumatic brain injury. NeuroRehabilitation. 2000; 15:107120.

13. Ponsford J. Sexual changes associated with traumatic brain injury. Neuropsychol Rehabil. 2003; 13:275-289.

14. Kreutzer JS, Zasler ND. Psychosexual consequences of traumatic brain injury: methodology and preliminary findings. Brain Inj. 1989; 3:177-186.

15 Blais M.C, Boisvert J.M. Psychological adjustment and marital satisfaction following head injury. Which critical personal characteristics should both partners develop. Brain Inj. 2007; 21:357-372.

16 Águila Martín, E .M; Sánchez Sánchez, L; Daza Gonzáles, M.T; La- calle Marcos, P; Fernández Agis, I. Disfunciones sexuales en pacientes con DCA: Estudio exploratorio descriptivo con el cuestionario EVAS-M y EVAS-H. Comunicación, Congreso Español de Sexología, V Encuentro Iberoamericano de Profesionales de la Sexología. 30 Sept-2 Oct. Santiago de Compostela. 2010

17 Sánchez Raja E., Honrubia Pérez, M. Estudio piloto sobre el efecto del TCE en la sexualidad del paciente. Mesa de Sexualidad y Discapacidad. XI Congreso Español de Sexología, V Encuentro Iberoamericano de Profesionales de la Sexología. 30 Sept-2 Oct. Santiago de Compostela. 2010 18 Marriner-Tomey A, Raile-Alligood M. Modelos y teorías en enfermería. $6^{\mathrm{a}}$ ed. Madrid: Elsevier; 2007.

19. Gordon M. Patrones funcionales. Madrid: Consejo Gral. de Enfermería. 20.Sánchez-Raja E, Honrubia-Pérez M, Chacón-Sánchez DD. Guía de educación afectivo sexual para personas con discapacidad visual. Barcelona: Universidad de Barcelona. 2007.

21. Fleck U. Sexual disturbances after brain injuries. Dtsch Med Wochenschr. $1952 ; 77: 139-141$.

22. Meyer JE. Sexual disorders in brain injured. Arch Psychiatr Nervenkr Z 
Gesamte Neurol Psychiatr. 1955; 193:449-469

23. Gray WG. Abnormal patterns of sexual behavior following brain trauma. Med Leg Bull. 1966; 15:1-6.

24. Oddy $M$, Humphrey $M$. Social recovery during the year following severe head injury. Journal of Neurology, Neurosurgery, and Psychiatry, 43, 798802. La recuperación social durante el año posterior a la lesión grave en la cabeza. J Neurol Neurosurg Psychiatry. 1980; 43:798-802

25. Gill CJ, Sander AM, Robins N, Mazzei DK, Struchen MA. Exploring experiences of intimacy from the viewpoint of individuals with traumatic brain injury and their partners. J Head Trauma Rehabil. 2011; 26:56-68.

26. Kosteljanetz, M. Jensen, T., Norgard, B., Lunde, I., Jensen, P., \& Johnsen, S. Sexual and hypothalamic dysfunction in the post-concussional syndrome. Acta Neurologica Scandinavica, 63, 169-80. La disfunción sexual y el hipotálamo en el síndrome post-conusional. Acta Neurológica Scandinavica. $1981 ; 63: 169-180$.

27. Simpson G, Long E. An evaluation of sex education and information resources and their provision to adults with traumatic brain injury. J Head Trauma Rehabil. 2004; 19:413-428. Kosteljanetz, M., Jensen, T., Norgard, B., Lunde, I., Jensen, P., \& Johnsen, S. (1981). 


\section{FIGURAS:}

Figura 1. Causas del Traumatismo Craneoencefálico

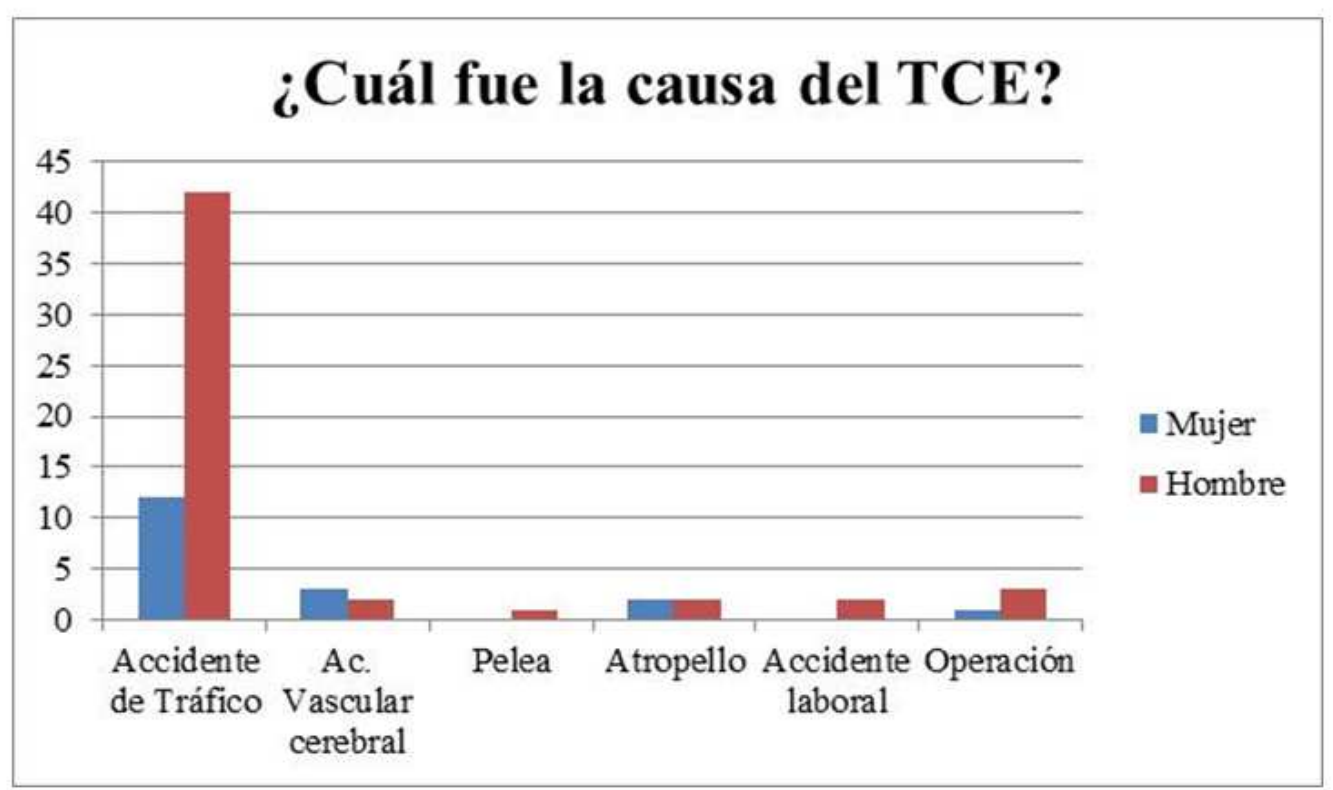


Figura 2. Calidad de las relaciones sexuales antes y después del TCE
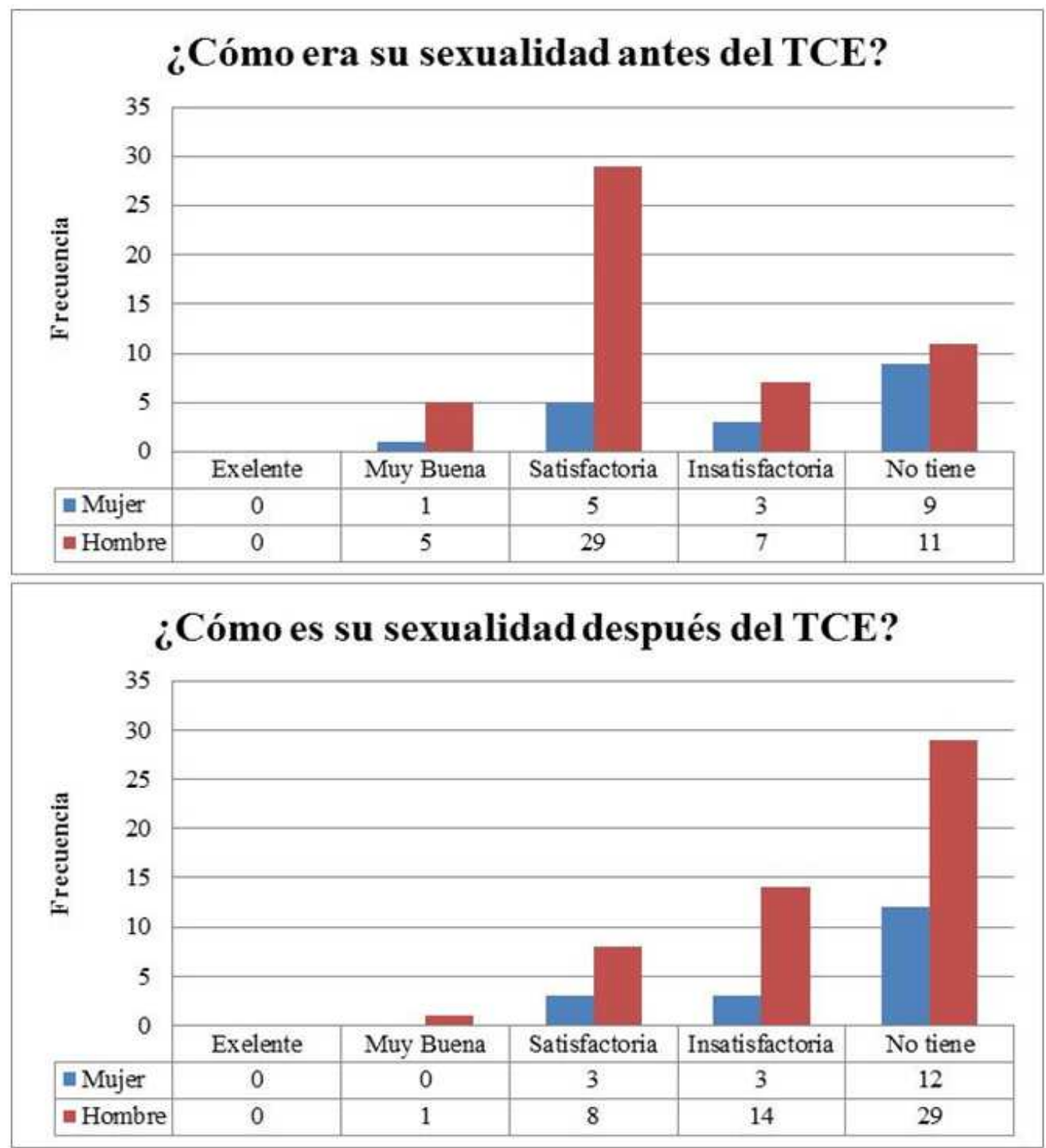
Figura 3. Actividad sexual en mujeres antes y después del TCE

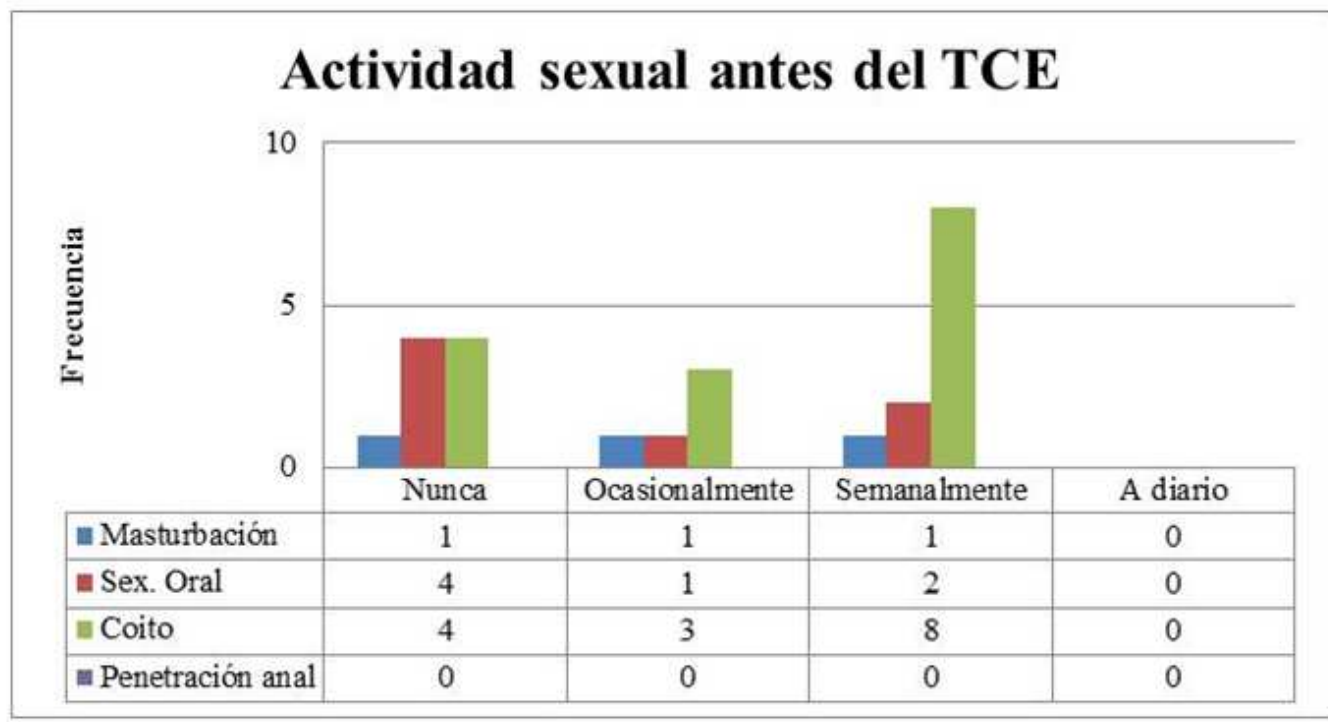

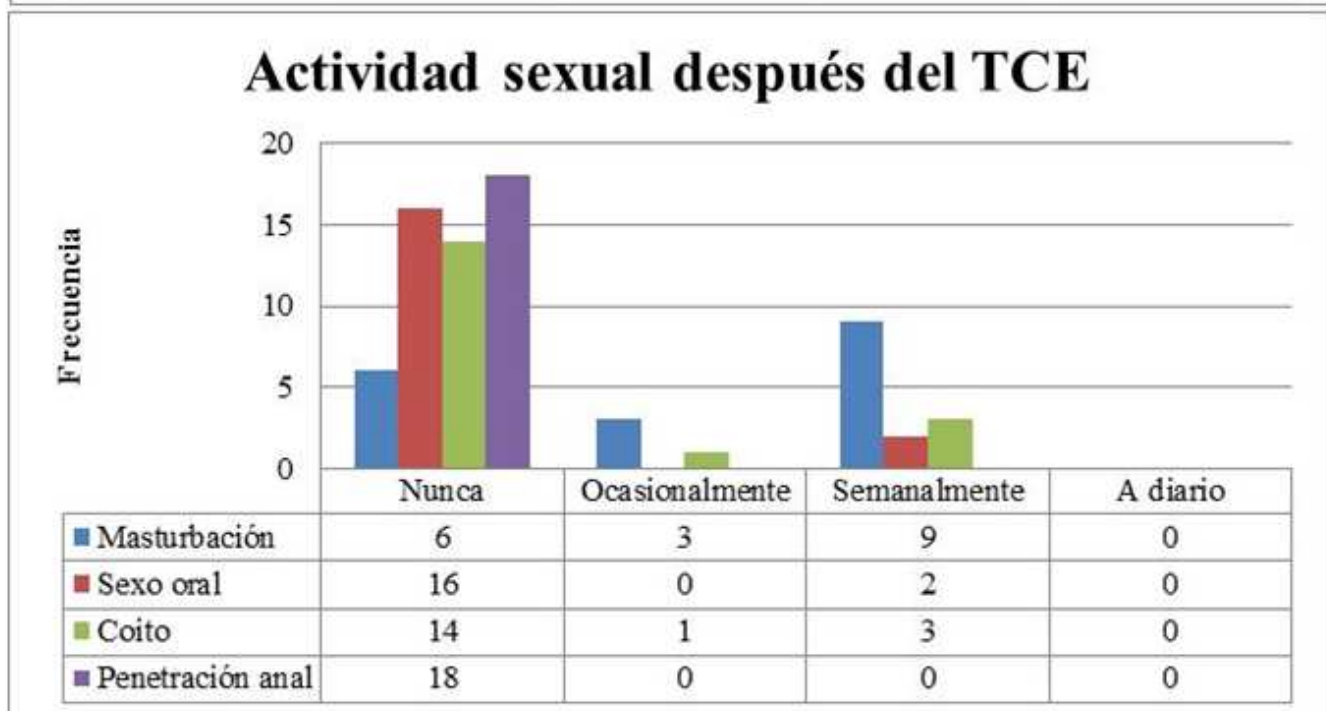


Figura 4. Actividad sexual en hombres antes y después del TCE
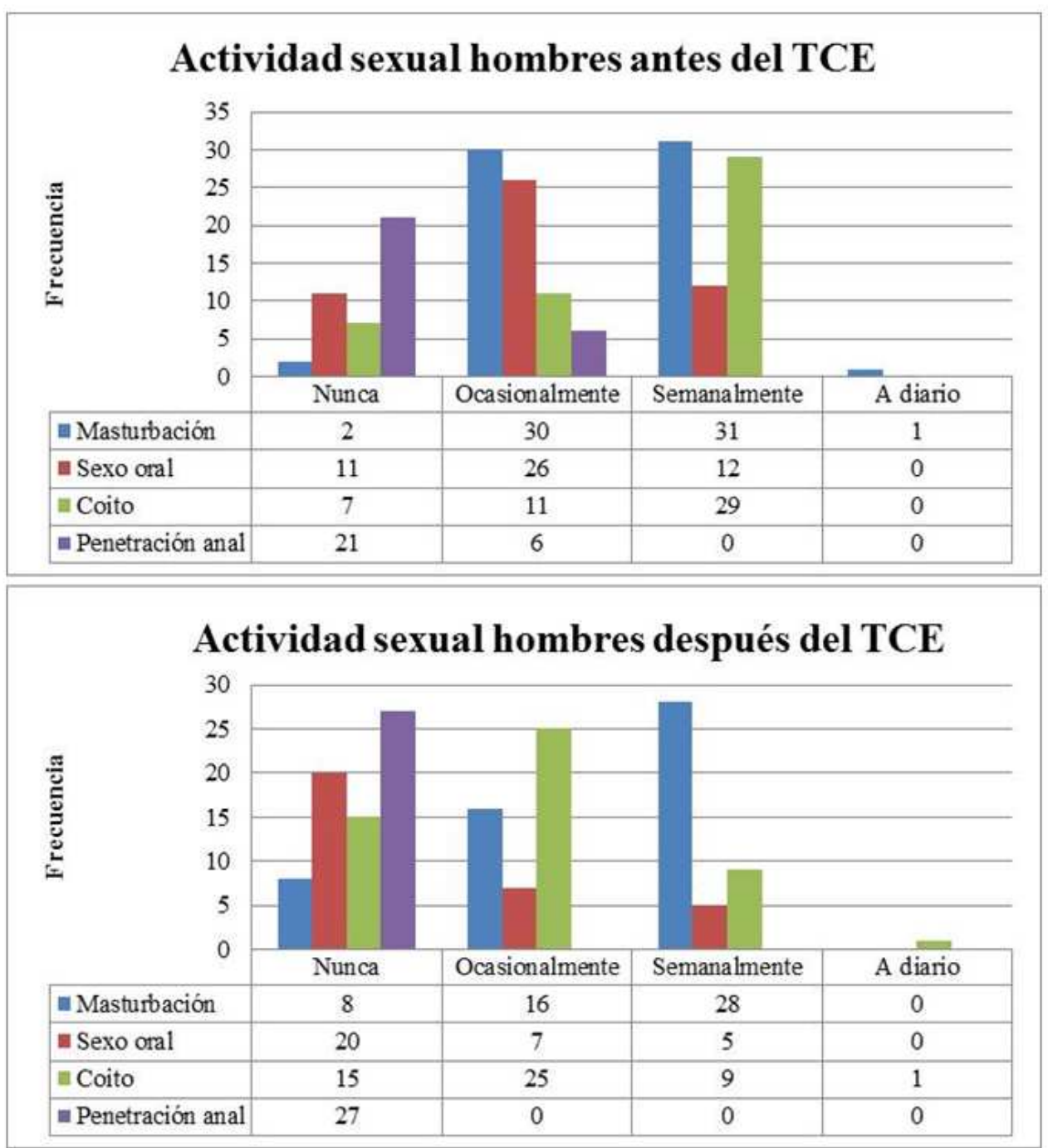